\title{
Revascularization-first strategy in acute aortic dissection with mesenteric malperfusion
}

\author{
Kayo Sugiyama ${ }^{1}$, Hirotaka Watanuki ${ }^{2}$, Masaho Okada ${ }^{3}$, Yasuhiro Futamura ${ }^{4}$, Rei \\ Wakayama $^{1}$, and Katsuhiko Matsuyama ${ }^{4}$ \\ ${ }^{1}$ Aichi Medical University \\ ${ }^{2}$ Aichi Ika Daigaku \\ ${ }^{3}$ Aichi Medical University School of Medicine \\ ${ }^{4}$ Aichi Medical University Hospital
}

June 22, 2020

\begin{abstract}
Background: Mesenteric malperfusion is a complication with a higher risk of in-hospital mortality because diagnosing mesenteric ischemia before necrotic change is difficult, and when it occurs, the patient's condition has worsened. Although it contradicts the previous consensus on central repair-first strategy, the revascularization-first strategy was found to be significantly associated with lower mortality rates. The aim of this study is to present our revascularization-first strategy and assess the postoperative results for acute aortic dissection involving mesenteric malperfusion. Methods: Among 58 patients with acute type A aortic dissection at our hospital between January 2017 and December 2019, mesenteric malperfusion was noted in six. Four hemodynamically stable patients underwent mesenteric revascularization with endovascular intervention in a hybrid operation room before central repair, and two hemodynamically unstable patients underwent central repair before mesenteric revascularization. Results: No in-hospital mortality was recorded. All four patients with mesenteric revascularization-first strategy recovered with no symptoms related to mesenteric ischemia. Two patients with central repair-first strategy developed paralytic ileus for 1 week; one of them needed explorative laparotomy, but no patients needed colon resection. Conclusion: The revascularization-first strategy in cases of acute type A aortic dissection with mesenteric malperfusion may achieve favorable results. However, in cases with other-major organ malperfusion or having hemodynamically unstable status, the appropriate strategy is controversial. Close evaluation of mesenteric perfusion using multiple modalities and prompt revascularization are mandatory in these complicated cases. A hybrid operation room provides an ideal environment for this revascularization-first strategy.
\end{abstract}

\section{MAIN TEXT}

\section{INTRODUCTION}

Over the past two decades, management of acute type A aortic dissection (AAD) has markedly improved. However, mesenteric malperfusion is an ominous complication carrying a higher risk of hospital mortality. ${ }^{1}$ Appropriate management of this fatal phenomenon remains controversial because of difficulty in obtaining accurate diagnosis and providing prompt treatment. ${ }^{2}{ }^{3}$ Previous reports proposed that central repair of entry closure and true lumen reinstallation should be performed first for early survival rate. ${ }^{4}$ However, recent results have reported inconsistent results. According to Kamman, surgical delay due to prioritizing release of ischemia is significantly associated with lower mortality rates. ${ }^{3}$ Deeb et al. has demonstrated the trend toward a better survival rate after endovascular perfusion repair prior to cardiopulmonary bypass. ${ }^{5}$ With these results, it is inferred that revascularization of mesenteric ischemia prior to definitive aortic repair may improve outcomes. In our institution, mesenteric revascularization with interventional radiology (IVR) precedes central aortic repair for hemodynamically stable patients. However, the appropriate strategy for hemody- 
namically unstable patients is still controversial. The aim of this study is to present our revascularization-first strategy and assess the postoperative results for AAD involving mesenteric malperfusion.

Hybrid operation rooms (hybrid ORs) are currently gaining popularity worldwide due to the exponential growth of endovascular aortic repair and transcatheter aortic valve implantation procedures. Hybrid ORs have enabled a variety of combined procedure including open surgical repair and endovascular treatment. Tsagakis et al. advocated the Hybrid OR concept to prioritize revascularization for ischemic organs even after cardiac drainage ${ }^{6,7}$ Because the infinite possibilities of the hybrid OR have been described, ${ }^{6,7}$ we also conducted this study to investigate the utility of the hybrid OR.

\section{MATERIALS AND METHODS}

\section{Study design}

This retrospective cohort study analyzed consecutive patients who underwent emergency surgical repair for AAD at Aichi Medical University Hospital from January 2017 to December 2019. Data were selected retrospectively from the institutional database for aortic disease.

Early and late clinical outcomes were assessed. Early outcomes included early mortality, cause of death, and mesenteric, cerebral, cardiac, respiratory, and renal complications. Late outcomes included late mortality, cause of death, major adverse aortic events (MAAE), and major adverse cardiac or cerebrovascular events. Mesenteric complication was a composite of mesenteric ischemic events and mesenteric reinterventions. Mesenteric ischemic events included paralytic ileus that needed fasting or intestine or colon resection. Mesenteric reinterventions included additional IVR or open revascularization for mesenteric ischemia. MAAE was a composite of either major aortic events or major aortic reinterventions. Major aortic events included rupture or re-dissection of the aorta. Major aortic reinterventions included additional thoracic endovascular aortic repair or a major surgical graft revision.

All procedures were performed in accordance with the Helsinki Declaration. The study was approved by the Ethics Committee of Aichi Medical University Hospital. All patients provided written consent that their clinical data might be used for scientific presentations or publications when they consulted the hospitals for the first time.

\section{Study population}

Diagnosis was confirmed using computed tomography (CT) in our hospital or other previous hospitals. Mesenteric malperfusion was defined as symptoms associated with mesenteric ischemia or severely impaired perfusion of the superior mesenteric artery (SMA) because of the extent of dissection. Mesenteric ischemia was characterized by abdominal symptoms including abdominal pain or vomiting, and the presence of metabolic acidosis including elevated lactate or decreased base excess. Demographics (age and sex), comorbidities (hypertension, chronic respiratory disease, chronic renal disease, diabetes mellitus, smoking history, and family history related to aortic dissection), and specific preoperative complications related to aortic dissection (other-organ malperfusion, aortic valve regurgitation, cardiac tamponade, shock hemodynamics, cardiac arrest, and resuscitation) were recorded. Revascularization for mesenteric ischemia was basically prioritized in cases with mesenteric malperfusion; however, in cases with hemodynamic instability due to cardiac tamponade or free rupture, central aortic repair was prioritized. In cases with other-major organ malperfusion, that is, cardiac or cerebral malperfusion, revascularization for these organs is preceded first. The priority of the strategy was discussed by the aortic team composed of surgeons, anesthesiologists, and radiologists.

\section{Revascularization and central repair procedure}

Catheter IVR for mesenteric malperfusion was performed by radiologists. Percutaneous access to the mesenteric artery was obtained from a common femoral artery approach using a 6- or 7-Fr sheath. Lesions were crossed with a 0.035 -inch, angle or straight, hydrophilic guidewire with or without a supporting 4-Fr straight catheter. Mesenteric lesions were treated with primary stenting using a self-expanding stent. Trans- 
esophageal echocardiography has been performed during IVR and central repair (Figure 1a); this modality enables evaluation of cardiac function, aortic valve regurgitation, and cardiac effusion, and moreover, it enables detection of whether the guidewire is placed in the true lumen or not during IVR.

Central aortic repair was performed through a median sternotomy using cardiopulmonary bypass with selective cerebral perfusion. Cardiopulmonary bypass was established with femoral artery and right axillar artery cannulation and bicaval venous drainage. After left ventricular venting, the patient was cooled down to $25^{\circ} \mathrm{C}$. Then aortic arch repair for entry closure and true lumen reinstallation was performed. The replacement range was determined depending on the position of the entry. When aortic dissection extended to the aortic root and detachment of the aortic valve commissure occurred, the valvular resuspension technique was adopted.

\section{RESULTS}

A total of 58 patients were admitted with AAD involving the ascending aorta. Of these patients, $6(10 \%)$ presented with mesenteric malperfusion. Baseline characteristics of these patients are presented in Table I. The mean age was 58 (range, 46-72) years, and 5 patients were men (83\%). All 6 patients showed static dissection in the SMA (Figure 2a, 2b), and 2 of them showed dissection in the celiac artery. Three patients complained of abdominal pain, and all 6 patients presented metabolic acidosis with elevated lactate and decreased base excess. History of current smoking was detected in all 6 patients, untreated hypertension was detected in 5 , chronic respiratory diseases including sleep apnea syndrome was detected in 3 , chronic kidney disease was found in 1 patient and paroxysmal atrial fibrillation was found in 1 patient. No patients had a family history of aortic dissection.

One patient had cerebral malperfusion, and 2 had moderate to severe aortic valve regurgitation. No patients had cardiopulmonary resuscitation and myocardial infarction; however, 2 patients presented with hemodynamic instability due to cardiac tamponade.

Operative procedures and outcomes are presented in Table II. Four patients underwent revascularizationfirst strategy in the hybrid OR, and 2 underwent central aortic repair prior to revascularization because of hemodynamic instability. As central aortic repair, total arch and hemiarch replacement were performed in 3 patients each. Pulmonary vein isolation was performed in 1 patient concomitantly. All patients were weaned from cardiopulmonary bypass uneventfully. After surgery, 2 patients with central repair-first strategy underwent IVR for mesenteric malperfusion. Explorative laparotomy (Figure 1b) was performed in 1 central repair-first patient because of prolonged metabolic acidosis during surgery. Because the small bowel was viable but poorly perfused entirely, the patient underwent prompt IVR with the chest open; as a result, colon resection was not required. Another patient underwent IVR immediately after central repair because of prolonged metabolic acidosis after surgery. After IVR, because the acidosis was improved to a normal range, colon resection was not required. All IVR procedures were performed successfully (Figure 3a, 3b). Endovascular stenting to the SMA was performed in all 6 patients, and stenting to celiac artery was performed in 1. All 4 patients with revascularization-first strategy recovered with no symptoms. On the contrary, 2 patients with central repair-first strategy developed paralytic ileus for 1 week (Figure 1c). No in-hospital mortality was recorded. Postoperative cerebral infarction occurred in 2 of central repair-first patients and tracheostomy was needed in them. Postoperatively, paroxysmal atrial fibrillation was observed in 2 patients and no patients had renal complications. During follow-up, MAAE occurred in 2 patients; additional thoracic endovascular aortic repair (1 patient) in 7 months and redo total arch replacement (1 patient) in 3 months were performed. Major adverse cardiac or cerebrovascular events occurred in 4 patients, of whom 3 developed stroke; 2 had a cerebral infarction during hospital days and the other had 3 months later, and 1 needed percutaneous catheter intervention in 10 months. One late mortality due to pneumonia was recorded in central repair-first patients.

\section{DISCUSSION}

The present study revealed that this revascularization-first strategy for mesenteric malperfusion was reasonable and feasible. Patients who underwent revascularization-first strategy recovered without any mesenteric 
ischemic events, whereas those who underwent central repair-first strategy developed paralytic ileus.

Despite improvement of in AAD management, organ malperfusion can be critical. ${ }^{1}$ Specific mesenteric malperfusion rates were reported between $1.4 \%$ and $8.2 \%{ }^{3}$ Among ischemic end-organ complications occurring at the onset of dissection, mesenteric malperfusion is one of the most insidious and challenging complications to manage. ${ }^{1,2}$

The classic treatment algorithm in AAD was to close the proximal entry tear and to reestablish peripheral true lumen perfusion. ${ }^{4,6,7}$ However, primary surgical repair of the entry site can add trauma to the alreadyischemic organs, and the ensuing uncontrolled reperfusion usually ends in patient death. Recent reports have reported paradoxical results. Kamman et al. described that surgical delay in malperfusion cases was significantly associated with lower mortality rates. ${ }^{3}$ They recommended relief of branch vessel obstruction first, followed by urgent aortic repair only when the malperfusion syndrome and its sequelae were resolved. ${ }^{3}$ Deeb et al. also recommended delaying surgery for primary distal reperfusion and stabilization. ${ }^{5}$ Tsagakis et al. proposed the hybrid OR concept prioritizing revascularization of malperfusion. ${ }^{6,7}$ Thus, the authors emphasized the controversial paradigm of mesenteric revascularization-first approach using IVR prior to the central repair to alleviate metabolic crisis.

Malperfusion has two types of pathophysiology, namely, dynamic and static. Dynamic malperfusion is defined as dissection with compression of the true lumen owing to false lumen pressurization with resulting flap occlusion of the orifice of the branch vessel. ${ }^{3}$ Static malperfusion is defined as dissection of the branch vessel with obstruction of the true lumen. In most cases, this is related to thrombosis of the false lumen with compression and obstruction of the true lumen. ${ }^{8}$ Static malperfusion may require initial intervention with stenting of the branch orifice or bypass for timely reperfusion. ${ }^{9}$ Previously, an aortic dissection with dynamic malperfusion may be treated with a central repair to entry closure. ${ }^{9}$ However, whether it is a dynamic or static malperfusion is usually unclear preoperatively. Furthermore, in many cases, a combination of a static and a dynamic component is present. ${ }^{3}$ Moreover, we should be cautious that malperfusion may worsen because the dissection flap extending into the branch changes after the central repair. ${ }^{9}$ Basically, in our institute, revascularization-first strategy is chosen for any type of malperfusion.

In accordance with Tsagakis et al., even in patients with initial circulatory instability due to tamponade, invasive diagnostics and treatment for organ malperfusion is possible in hybrid OR after cautious tamponade release and pericardial drainage under blood pressure control. ${ }^{6,7}$ Tsagakis et al. have proposed that revascularization of malperfusion should be considered after cardiac drainage in hybrid OR even after full sternotomy. ${ }^{6}{ }^{7}$ In the present study, central repair was prioritized in two cases because they were hemodynamically unstable. Even in hemodynamically unstable cases, immediate tamponade release via median sternotomy or cautious pericardial drainage placement should have been considered. After attaining hemodynamic stability, IVR should have been prioritized, followed by central repair. However, in a hemodynamically compromised case with free rupture or cardiopulmonary resuscitation, central aortic repair is inevitable. Moreover, in a case with cerebral or cardiac malperfusion, revascularization for them should be performed first. Therefore, the strategy for these severely complicated cases is still controversial.

In the 1990s, percutaneous techniques were introduced to restore end-organ perfusion and reduce morbidity associated with open surgical repair in a complex and high-risk patient cohort of AAD. ${ }^{3}$ This approach consisted of flap fenestration and placement of a true lumen stent in the visceral artery to eliminate dynamic obstruction. IVR was less invasive than open surgical revascularization for mesenteric malperfusion.

Hybrid ORs are currently gaining popularity worldwide due to the exponential growth of transcatheter aortic valve implantation procedures. Tsagakis et al. recommended that all patients with established or suspected diagnosis of acute aortic syndrome be admitted directly from helicopter or ambulance transport to the Hybrid OR in the presence of aortic team. ${ }^{6,7}$ The potential of this hybrid OR concept is that it enables fast online diagnostics, followed by immediate intervention and/or open surgery, particularly for hemodynamically unstable patients. ${ }^{6,7}$ In our institute, all patients from helicopter or ambulance transport suspected of acute aortic syndrome are checked at the emergency room before transport to the hybrid OR. 
The hybrid OR concept enables us to eliminate time loss and represents the ideal environment for the aortic team composed of surgeons, radiologists, and cardiac anesthesiologists. Direct transport to the hybrid OR should be incorporated in our institute.

Our study is limited by several factors. First, a small number of patients were enrolled owing to the rarity of the condition. Second, it was a retrospective, single-center experience that lacked any form of randomization. Finally, the surgical technique for AAD has evolved during the time period of this study. In the future, multicenter study will be necessary to compensate for these limitations. Furthermore, global research not only domestic, should be conducted. On the other hand, in our institute, the hybrid OR concept proposed by Tsagakis et al. should be promoted. The better performance of aortic team is expected in the treatment of acute aortic syndrome.

\section{CONCLUSIONS}

The revascularization-first strategy in AAD with mesenteric malperfusion was acceptable and may achieve favorable results. However, the appropriate strategy for severely complicated cases, such as those with other-major organ malperfusion or having hemodynamically unstable status, remains controversial. Close evaluation of mesenteric perfusion using multiple modalities and prompt revascularization are mandatory in these complicated cases. Moreover, IVR at the hybrid OR enables immediate revascularization, followed by central aortic repair. A hybrid OR provides an ideal environment where interventional radiology and surgery can be performed at the same time for the aortic team of cardiac surgeons, radiologists, and anesthesiologists.

Acknowledgements We thank the Honyaku Center for reviewing and editing the manuscript. We also thank our colleagues for their helpful comments.

Disclosure Statement There are no conflicts of interest to declare.

Funding statement: We did not receive any financial support for this study.

\section{REFERENCES}

1. Orihashi K. Mesenteric ischemia in acute aortic dissection. General Thoracic and Cardiovascular Surgery. 2018; DOI: 10.1007/s11748-018-0970-6

2. Di Eusanio M, Trimarchi S, Patel HJ, Hutchison S, Suzuki T, Peterson MD. Clinical presentation, management, and short-term outcome of patients with type A acute dissection complicated by mesenteric malperfusion: observations from the International Registry of Acute Aortic Dissection. J Thorac Cardiovasc Surg. 2013; 145: 385-390.

3. Kamman AV, Yang B, Kim KM, Williams DM, Michael Deeb G, Patel HJ. Visceral Malperfusion in Aortic Dissection: The Michigan Experience. Semin Thorac Cardiovasc Surg. 2017; 29: 173-178.

4. Hagan PG, Nienaber CA, Isselbacher EM, Bruckman D, Karavite DJ, Russman PL. The international registry of acute aortic dissection (IRAD). New insights into an old disease. JAMA. 2000; 283: 897-903.

5. Deeb GM, Williams DM, Bolling SF, Quint LE, Monaghan H, Sievers J. Surgical delay for acute type A dissection with malperfusion. Ann Thorac Surg. 1997; 64: 1669-75; discussion 1675-7.

6. Tsagakis K, Konorza T, Dohle DS, Kottenberg E, Buck T, Thielmann M. Hybrid operating room concept for combined diagnostics, intervention and surgery in acute type A dissection. Eur J Cardiothorac Surg. 2013; 43: 397-404.

7. Tsagakis K, Jánosi RA, Frey UH, Schlosser T, Chiesa R, Rassaf T. True Lumen Stabilization to Overcome Malperfusion in Acute Type I Aortic Dissection. Semin Thorac Cardiovasc Surg. 2019; 1: 740-748.

8. Yang B, Patel HJ, Williams DM, Dasika NL, Deeb GM. Management of type A dissection with malperfusion. Annals of Cardiothoracic Surgery. 2016; 5: 265-274.

9. Tanaka A, Estrera AL. Mesenteric Malperfusion: The Insidious, Dreadful Enemy. Semin Thorac Cardiovasc Surg. 2017; 29: 179-180.

Figure legends 
Figure 1a Transesophageal echocardiography performed continuously in the hybrid operation room

Figure 1b Intraoperative view showing insufficient blood perfusion in the entire intestine during explorative laparotomy

Figure 1c Abdominal radiograph showing paralytic ileus

Figure 2a, 2b Preoperative computed tomographic image showing severely impaired perfusion of the superior mesenteric artery because of the extent of dissection.

Figure 3a Angiogram before revascularization showing poor perfusion in the superior mesenteric artery

Figure 3b Angiogram after revascularization showing improvement of perfusion in the superior mesenteric artery

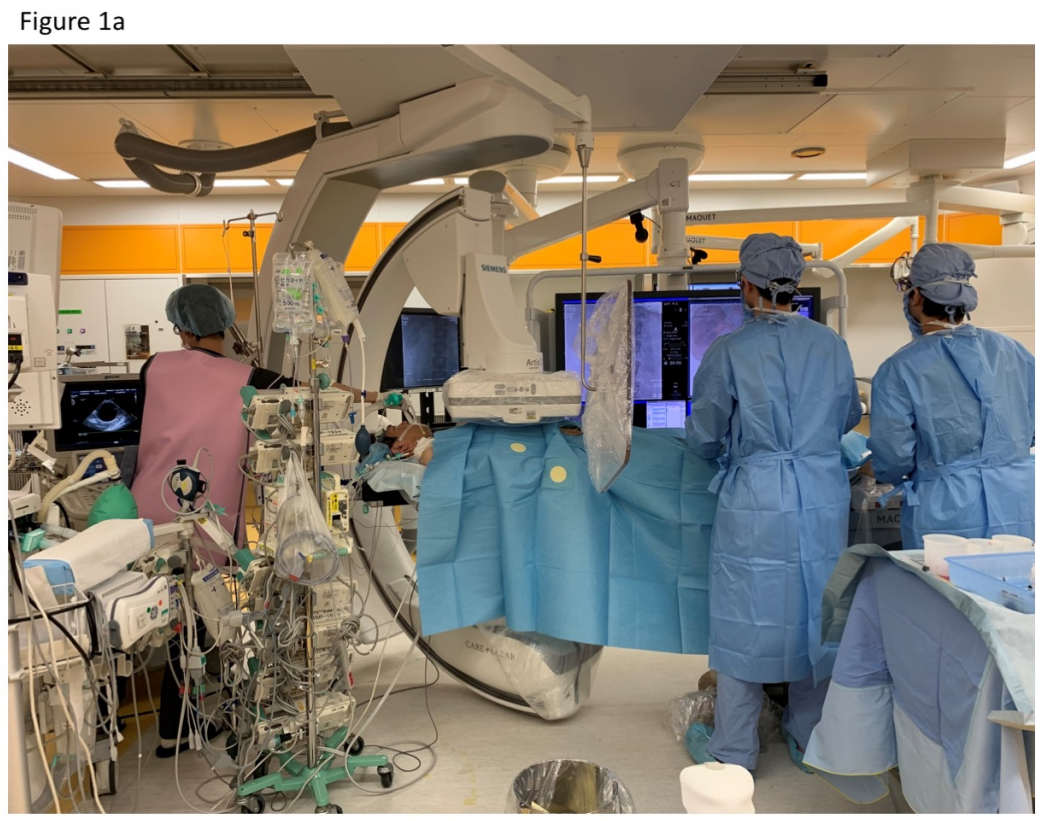


Figure $1 b$

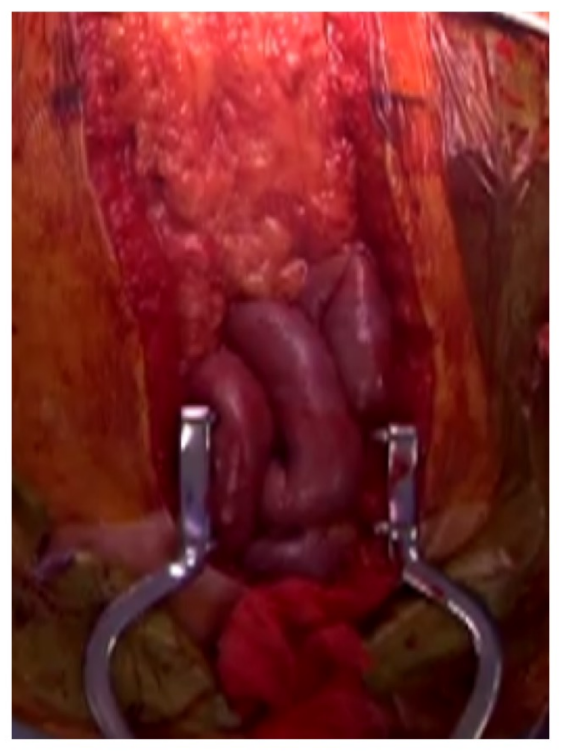

Figure 1c

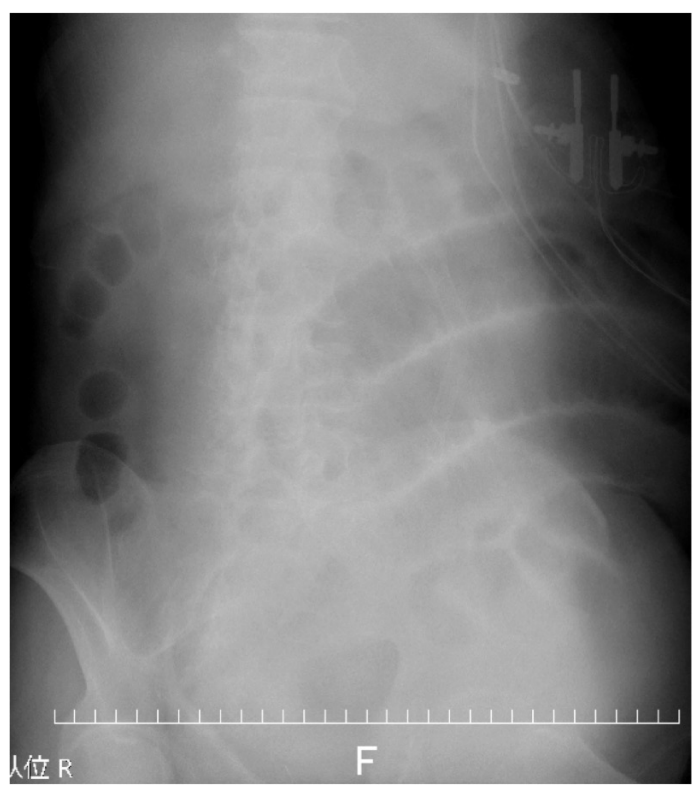


Figure 2a

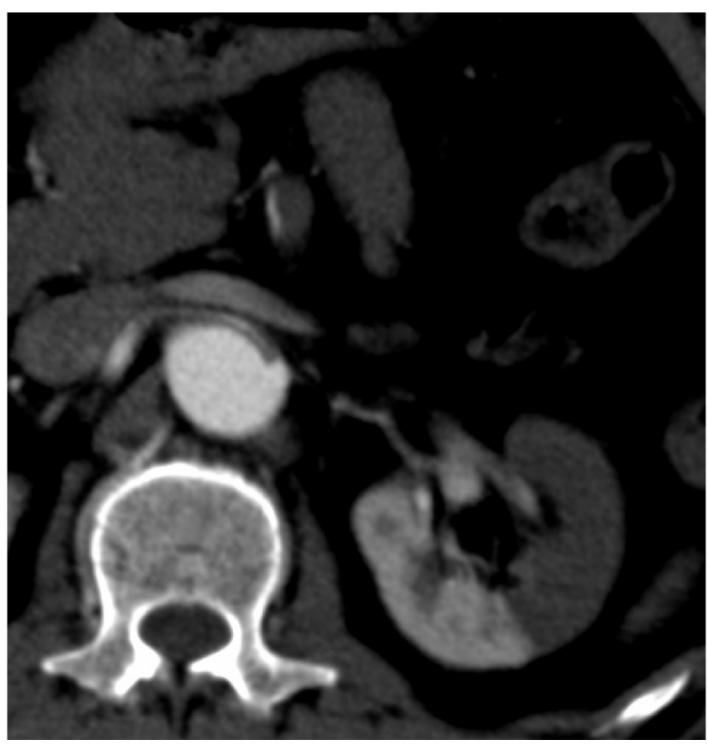

Figure $2 b$

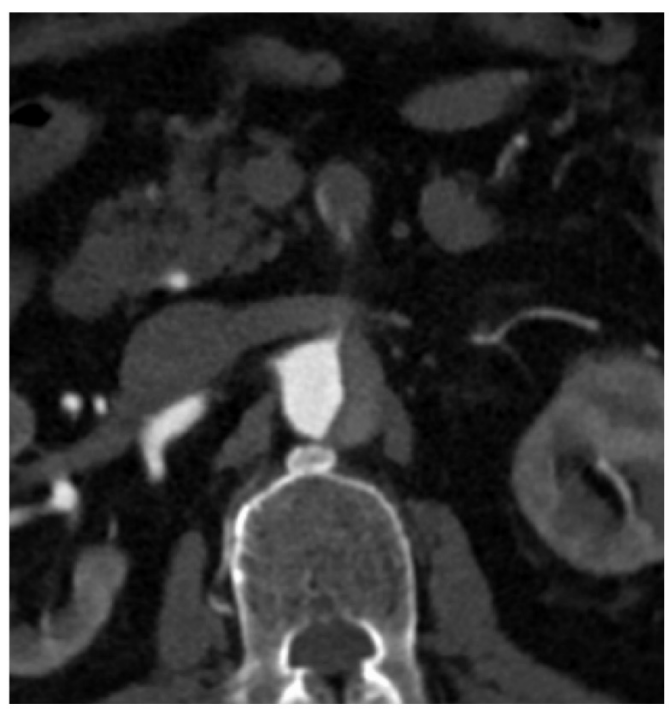


Figure 3a

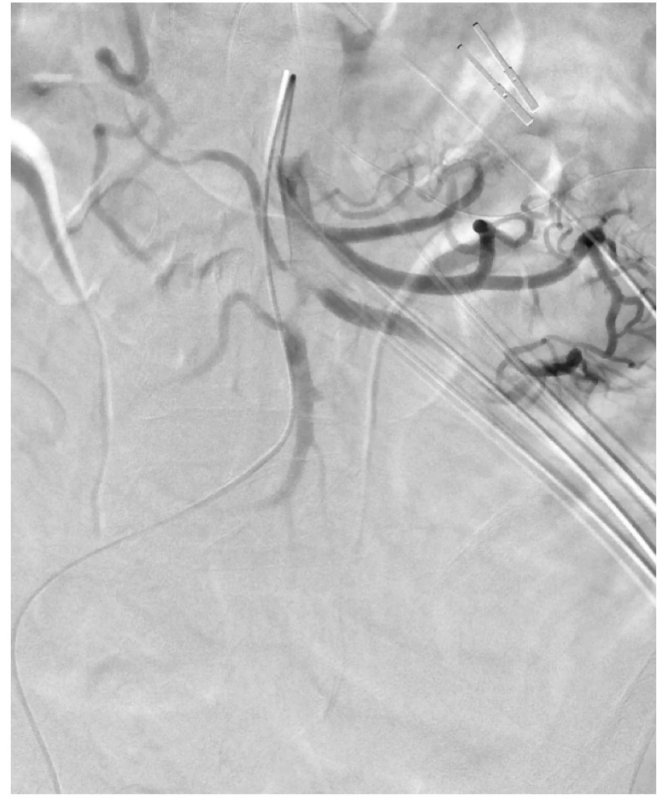

Figure $3 b$

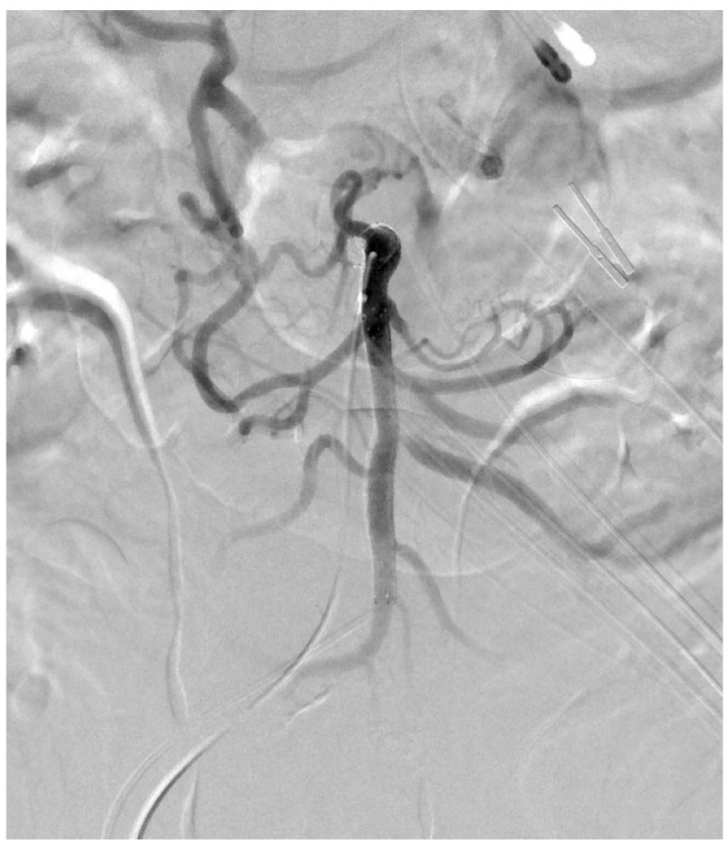


Table 1 Characteristics of patients undergoing surgical intervention for acute aortic dissection with mesenteric malperfusion

\begin{tabular}{|c|c|c|c|c|c|c|}
\hline patient & 1 & 2 & 3 & 4 & 5 & 6 \\
\hline Age $(y)$ & 46 & 69 & 60 & 72 & 48 & 56 \\
\hline Sex & $\mathrm{m}$ & $\mathrm{m}$ & $\mathrm{m}$ & f & $\mathrm{m}$ & $\mathrm{m}$ \\
\hline Comorbidities & HT, SAS, smoking & $H T$, CRD, smoking & HT, CKD, smoking & Paf, smoking & $\begin{array}{l}\text { HT, DM, CRD, } \\
\text { smoking }\end{array}$ & $H T$, smoking \\
\hline Family history & none & none & none & none & none & none \\
\hline Abdominal symptom & abdominal pain & abdominal pain & none & vomit & none & none \\
\hline Acidosis & yes & yes & yes & yes & yes & yes \\
\hline Other malperfusion & none & yes (brain) & none & none & none & none \\
\hline Aortic valve regurgitation & yes & none & none & none & none & yes \\
\hline Cardiac tamponade & none & yes & none & none & none & yes \\
\hline Shock hemodynamics & yes & yes & none & none & none & none \\
\hline Cardiac arrest, resuscitation & none & none & none & none & none & none \\
\hline
\end{tabular}

Table 2 Operative procedures and outcomes

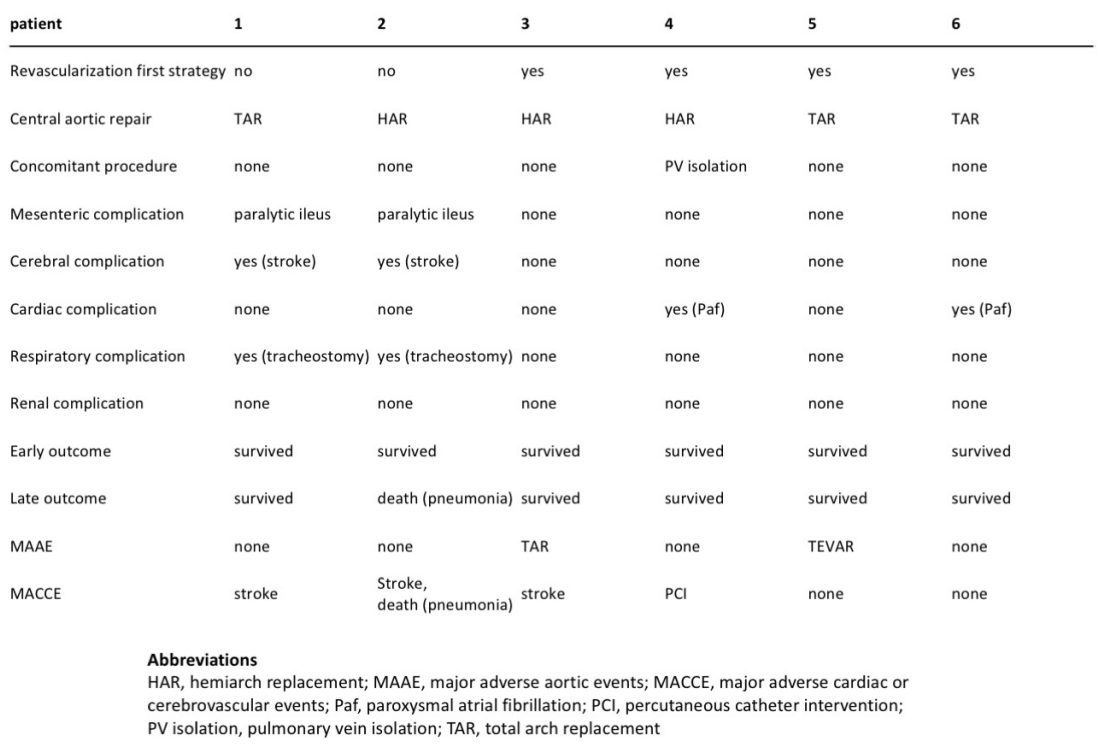

